Meta

Journal des traducteurs

Translators' Journal

\title{
Le chemin des écoliers
}

\section{Ana Peyrelongue}

Volume 35, numéro 3, septembre 1990

La traduction dans le monde hispanolusophone

URI : https://id.erudit.org/iderudit/003831ar

DOI : https://doi.org/10.7202/003831ar

Aller au sommaire du numéro

Éditeur(s)

Les Presses de l'Université de Montréal

ISSN

0026-0452 (imprimé)

1492-1421 (numérique)

Découvrir la revue

Citer cet article

Peyrelongue, A. (1990). Le chemin des écoliers. Meta, 35(3), 625-631.

https://doi.org/10.7202/003831ar

Ce document est protégé par la loi sur le droit d'auteur. L'utilisation des services d'Érudit (y compris la reproduction) est assujettie à sa politique d'utilisation que vous pouvez consulter en ligne.

https://apropos.erudit.org/fr/usagers/politique-dutilisation/
Cet article est diffusé et préservé par Érudit.

Érudit est un consortium interuniversitaire sans but lucratif composé de l’Université de Montréal, l'Université Laval et l'Université du Québec à Montréal. Il a pour mission la promotion et la valorisation de la recherche. https://www.erudit.org/fr/ 


\section{LE CHEMIN DES ÉCOLIERS}

Ana Peyrelongue

Santiago de Tepetlapa, Mexico

Lorsqu'on m'a demandé de décrire mon expérience de traductrice, je me suis aperçue qu'il ne m'était pas arrivé de consacrer à la réflexion sur cette activité, qui occupait pourtant de longues heures de ma journée, le temps qu'elle méritait. Mais c'est justement parce que c'était une occasion privilégiee de réparer ma négligence que j'ai accepté de le faire même au risque de dire des banalités.

Hispanophone, professeur de langue et littérature françaises, je considérais la version comme un exercice pédagogique très favorable certes, mais à l'acquisition de la mâ̂trise de la langue maternelle. Écrire d'après les mots des maîtres est, en effet, une pratique enrichissante pour celui qui s'y livre mais qui n'exige pas une connaissance active de la langue étrangère.

Conduite par les circonstances au Mexique, un pays où, en raison de sa proximité des États-Unis, l'anglais est la première - sinon la seule — langue étrangère obligatoire dans les programmes scolaires, je fis mes premiers pas comme traductrice temporaire. Plus tard, $\mathrm{j}$ 'ai été invitée à enseigner la littérature française - en traduction - à des élèves qui ne connaissaient pas le français. Il s'agissait de séminaires de diffusion culturelle destinés à des étudiants qui préparaient leur licence d'histoire de l'art ou de philosophie dans un collège de province. Expérience tout à fait nouvelle pour une enseignante nourrie d'une orthodoxie pédagogique qui condamnait le recours à la langue maternelle en classe de français. Heureusement, on me laissait toute liberté de choisir le sujet de mes cours. $\mathrm{Si}$ au premier abord la proposition me semblait sacrilège, réflexion faite, elle n'avait rien de bizarre. Du point de vue linguistique, l'élève étranger et le national sont à peu près dans la même situation quand il s'agit d'apprécier un texte littéraire, notamment lorsqu'il est question des cuvres du passé. Au fond, lire un texte littéraire c'est l'expliquer, le commenter, même si on le lit dans sa propre langue, celle de l'écrivain n'étant pas toujours accessible à tous. D'ailleurs, une bonne traduction, même si elle ne réussit pas à tout rendre, permet de faire passer le «poème». «Après tout», dit Marguerite Yourcenar, grand écrivain et traductrice expérimentée d'œuvres du présent et du passé, «les trois quarts de ce que nous lisons est traduction. Nous lisons la Bible en traduction, les poètes chinois, les poètes japonais, les poètes hindous, Shakespeare quand on ne sait pas l'anglais, Goethe quand on ne sait pas l'allemand. On serait très limité si on ne disposait pas de traductions ${ }^{1}$.» C'est vrai, le lecteur polyglotte est l'exception.

J'ai commencé alors par l'étude des œuvres contemporaines pour lesquelles on disposait d'excellentes traductions en espagnol. Le discours organique de M. Duras et le discours organisé de $M$. Yourcenar ont très bien passé l'épreuve. En comparant le texte original avec sa traduction, pendant la préparation de mes cours, j'ai pénétré bien des secrets de cet art injustement décrié et j'ai appris à le revaloriser. Cette expérience m'encouragea aussi à dépoussiérer des traductions que j'avais entreprises pour mon plaisir sans aucun souci de publication et qui dormaient oubliées au fond d'un tiroir. Á la recherche du discours féminin dans la littérature française, $\mathrm{j}$ 'avais trouvé l'amour et la folie dans l'œuvre de Louise Labé et la dialectique du couple dans les Lettres d' Hélö̈se et d'Abélard. Je m'étais aperçue que ce travail pourrait être utile non seulement à mes 
élèves. Les traductions de livres anciens devenaient de plus en plus rares. Il me semblait que l'ignorance du passé et le mépris qui en résulte, loin d'accélérer le progrès, avaient ralenti cette «ascension de la brute vers l'homme» en faisant de nous des êtres sans mémoire, intoxiqués de sexe, de violence et de mercantilisme. Une promenade dans la littérature ancienne nous permettrait de revisiter ce passé, non pas pour y aller chercher l'avenir, certes, mais pour récupérer ce qu'il a de récupérable. Décidée à partager ma découverte, j'ai songé à remanier mon travail pour le rendre publiable.

Il me faut avouer que je n'ai pas fréquenté les centres de formation spécialisés pour traducteurs. Intuitive, artisanale, ignorante de toute théorie, je me suis lancée dans cette entreprise pour l'amour de la littérature plutôt que pour remplir un devoir professionnel. Je suis donc arrivée à la traduction par le chemin des écoliers, et c'est pour ne pas trahir la confiance qu'on m'a donnée en sollicitant ma collaboration pour ce numéro et parce que j'avais besoin de guides pour revenir sur mes pas que je me suis appliquée à combler mes lacunes théoriques. C'était d'ailleurs pour moi l'opportunité de me familiariser avec la lecture des spécialistes de l'«opération traduisante». Lecture bénéfique qui m'a éclairée sur ce métier qui était devenu le mien. C'est ainsi que j'ai appris que la traduction orale et écrite est aussi ancienne que la parole et l'écriture; qu'il y a trois mille ans, les traducteurs de la cour des pharaons avaient le rang de princes; qu'aujourd'hui ils ont celui de galériens; qu'on essaie de les libérer de leurs chaînes en les supplantant par des machines électroniques, etc. Mais M. Jourdain apprenant qu'il parlait en prose n'était pas plus étonné que moi lorsque je découvris que je traduisais selon les règles, et que le lot du traducteur était de ne jamais échapper au sentiment d'impuissance devant l'intraduisibilité et au complexe de culpabilité devant l'auteur qui en est la résultante. Une connaissance préalable des ouvrages théoriques m'aurait sans doute épargné bien des tâtonnements.

Mais je reviens à mon sujet... Essayer de familiariser un lecteur trop installé dans la modernité locale avec les idées et la sensibilité d'une époque et d'un monde lointains n'est pas chose facile. Il m'a fallu improviser une méthode de travail. Une première traduction, littérale, m'avait mise en face d'un échantillon de problèmes que j'aurais à résoudre pour ne pas trahir le message que je prétendais transmettre. Le plus urgent, à mon avis, était celui de choisir ce que les théoriciens appellent le registre de translation. Imiter la langue de Góngora ou celle de Cervantes pour traduire le Débat de Folie et d'Amour, ou celle du Poème du Cid pour traduire les Lettres d'Héloïse et d'Abélard contrariait mon dessein de mettre en valeur la modernité de l'Antiquité. C'était surtout le Débat... qui me mettait dans ce dilemme, quant aux Lettres..., le choix avait déjà été fait, comme on verra plus loin. Je ne voulais pas rebuter le lecteur en le mettant dans l'embarras d'avoir à traduire ma traduction. Au contraire, $\mathrm{j}$ 'aspirais à lui faire sentir combien le discours de Louise Labé ou celui d'Héloüse sont proches de nous, mais sans les décolorer. J'éprouvais le besoin de m'imprégner de mes auteurs, de plonger dans leurs vies, leurs œuvres, leurs mondes. Je m'adonnais donc aux recherches et aux annotations pertinentes. D'une part, je retrouvais l'atmosphère, d'autre part, les notes prises pour mon usage serviraient plus tard à renseigner le lecteur. Mon intuition première se confirmait au fur et à mesure que j'avançais dans ce sens: l'espagnol d'aujourd'hui laisserait transparaître l'étrangeté du texte original, son exotisme, pour ainsi dire. En effet, la plupart des mots pour l'exprimer (les faits de civilisation, par exemple) sont tombés en désuétude sans être absolument écartés de l'usage normal; en français comme en espagnol ils font partie de ce qu'on appelle le vocabulaire passif. Le voisinage des langues tournait, sur ce point, à mon avantage. Ce travail préparatoire me permit de dissiper mes doutes: le pastiche n'étant pas de mon domaine, les raisons que je viens de signaler et le but que je m'étais proposé orientaient ma décision vers l'emploi de l'espagnol moderne tout en respectant, bien entendu, le style soutenu de l'original. Ce premier pas effectué, je repris mon 
premier mot à mot pour le défaire et le refaire autant de fois qu'il me parut nécessaire pour réussir — naiveté suprême ! - le parfait mariage de la beauté et de la fidélité. Heureusement, je n'étais pas payée à la page !

Les difficultés se présentèrent dès le premier coup d'œil jeté sur l'œuvre à traduire. S'il n'existait pas de transcriptions des textes anciens, on aurait, en plus, le travail de décryptage de la calligraphie - par exemple, les caractères droits à angles et à crochets de l'écriture gothique des premières éditions des œuvres de Louise Labé. Puis, l'extrême liberté orthographique et syntaxique du français du XVIe siècle se heurtait parfois à la sévère codification de l'espagnol contemporain. Il s'agissait de traduire une langue qui n'avait pas encore une configuration définitive, donc adolescente, en une autre, mûre, adulte, ce qui n'est pas toujours avantageux pour le traducteur. Il risque, à chaque instant, de tomber dans la tentation de la facilité offerte par le maniement d'un système d'expression plus évolué, et de dénaturer ainsi les paroles pleines de fraîcheur, de cette naìveté apparente qui caractérise les textes de cette époque. Je ne suis pas sûre d'y avoir bien résisté.

Voilà, à titre d'illustration, un exemple de la «syntaxe escarpée et des aspérités

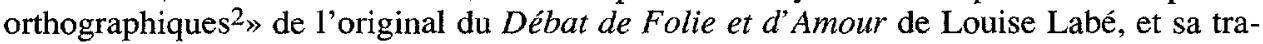
duction en espagnol. L'absence de règles fixxes d'écriture donne au langage une malléabilité dont la poétesse a su profiter et qui n'est pas toujours facile à rendre.

Exprimez tant que voudrez la force d'un oil; faites le tirer mil traits par jour; n'oubliez qu'une ligne qui passe par le milieu, jointe avec le sourcil, est un vrai arc: que ce petit humide que l'on voit luire au milieu, est le trait prest à partir; si est ce que toutes ces flesches n'iront en autres cœurs, que ceus que Folie aura preparez. Que tant de grans personnages, qui ont esté et sont de present, ne s'estiment estre injuriez, si pour avoir aymé je les nome fols. Qu'ils se prennent a leurs Filozofes, qui ont estimé Folie estre privacion de sagesse, et sagesse estre sans passions: desquelle Amour ne sera non plus destitué, que la Mer d'ondes et vagues: vray est qu'aucuns dissimulent mieus leur passion : et s'ils s'en trouvent mal, c'est une autre espèce de folie. Mais ceus qui montrent leurs afeccions estans plus grandes que les secrets de leurs poitrines, vous rendront et exprimeront une si vive image de Folie, qu'Apelle ne la sauroit mieus tirer au vif... Et penseriez vous, que les amours des femmes soient de beaucoup plus sages? les plus sages se laient bruler dedens le corp avant que de rien avouer. Et combien qu'elles vouissent prier, si elles osoient, elles se laissent adorer : et toujours refusent ce qu'elles voudroient bien que lon leur ostat par force...

Decid cuanto queráis de la fuerza del ojo, hacedle lanzar mil flechas al dia; no olvidéis que una línea que pasa por el medio, unida a la ceja, forma un verdadero arco, que esa pequeña humedad que se ve brillar al centro, es la flecha lista para partir; sin embargo todas esas flechas sólo irán a dar a aquéllos corazones que Locura haya preparado. Que tantos grandes personajes que existieron y que aún existen no se sientan injuriados si los llamo locos por haber amado. Que culpen a sus filosofos que consideraron a la locura como una privación de cordura, y a la cordura una carencia de pasiones, sin las cuales el amor dejaría de existir, como el mar sin sus ondas y sus olas. Cierto es que algunos disimulan mejor sus pasiones; y si sufren es otra especie de Locura. Pero aquéllos que ostentan su cariño por ser mayor que los secretos de sus pechos, os entregarán y expresarán una imagen tan viva de la locura que Apeles no podría reproducirla mejor.. Quinzás penséis que los amores de las mujeres son mucho más cuerdos. Las más cuerdas se dejarían quemar por dentro del cuerpo antes de confesar nada. Y aunque quisieran rogar, si se atrevieran, se dejan adorar ; y siempre niegan aquéllo que les gustaria que les quitaran por la fuerza...

Le texte original permet d'apprécier la liberté orthographique et syntaxique dont on a parlé plus haut. L'emploi de $s, z, i, y$, par exemple n'est pas encore fixé. Il en est de même pour les accents. L'accent aigu est assez fréquent au XVI' siècle, mais l'emploi des accents grave et circonflexe s'est répandu beaucoup plus tard. En effet, c'est seulement 
au XVIII ${ }^{e}$ siècle que l'accent circonflexe est venu remplacer le $\mathbf{s}$ d'allongement ou les lettres disparues; l'orthographe des terminaisons de l'imparfait et du conditionnel (ois - oit - oient) subsiste jusqu'au début du XIX ${ }^{\mathrm{e}}$ siècle. L'emploi des majuscules, la ponctuation, l'omission du pronom personnel sujet, l'ordre des pronoms personnels compléments dans la phrase, etc. sont autant de particularités du français moyen qui peuvent devenir des pièges pour un traducteur. Mais je ne dois pas m'attarder sur ce point qui m'éloignerait de mon but et qui, de plus, ferait l'objet d'un traité de grammaire diachronique.

Quant à mes Cartas de Abelardo y de Eloísa, elles sont la traduction d'une traduction. Écrites en latin au XII siècle, elles ont été traduites en français pour la première fois par Jean de Meung au XIII e siècle. Réunies et publiées comme un livre selon un manuscrit latin trouvé dans la bibliothèque de François d'Amboise, au XVe siècle, elles ont connu une popularité immense grâce à leurs nombreuses traductions et adaptations. Leur véritable texte, rétabli par Victor Cousin au XIXe siècle, encouragea les latinistes de l'époque à entreprendre des versions plus fidèles et plus complètes du recueil. Parmi celles-ci, $\mathrm{j}^{\prime}$ ai eu le coup de foudre pour celle d'Octave Gréard 3 , publiée une première fois en édition bilingue en 1869 et puis, seule, en plusieurs éditions successives jusqu'en 1925. Je n'ai pas pourtant négligé les autres, c'est-à-dire celles dont je pouvais disposer pour les comparer: deux françaises, des dix-huitième et dix-neuvième siècles respectivement; deux espagnoles, sans date. Mais la traduction d'O. Gréard est devenue mon texte de base. J'appris au cours de mon enquête qu'au dire des critiques c'était la meilleure.

Cette fois mon expérience antérieure a facilité ma tâche. Et à vrai dire, je traduisais par personne interposée, mes connaissances rudimentaires du latin ne me permettaient pas d'agir autrement. $O$. Gréard s'est chargé de déblayer mon chemin; je lui en sais gré. C'est lui qui évita les écueils de la syntaxe latine - du XII ${ }^{\mathrm{e}}$ siècle - et qui s'évertua à rendre, par exemple, les raccourcis, intraduisibles parfois, si adroitement employés par Hélö̈se pour les suscriptions de ses lettres.

Suivant l'esprit de l'époque, Hélöise commence ses lettres par une formule quasi télégraphique résumant leur contenu. Nous donnons ensuite le texte original et les traductions française et espagnole de ces formules.

L'en-tête de sa première lettre exprime tout ce qu'Abélard a été pour elle, tout ce qu'elle a été pour lui. En effet, celui-ci fut successivement son maître et amant, son époux, et après leur conversion, son père spirituel et son frère en Jésus-Christ. Voilà en quelques mots toute l'histoire de ce couple.

Latin: Domino suo, imo patri; conjugi suo, imo fratri; ancilla sua, imo filia; ipsius uxor, imo soror; Abelardo Heloissa.

Français : A son maitre ou plutôt à son père; à son époux, ou plutôt à son frère; sa servante, ou plutôt sa fille; son épouse ou plutôt sa sœur; à Abélard, Héloïse.

Espagnol: A su amo, o mejor dicho, a su padre; a su esposo, o mejor dicho, a su hermano; su sierva, o mejor dicho, su hija; su esposa, o mejor dicho, su hermana; a Abelardo, Eloísa.

Il faut noter qu'en français maître a les deux sens de seigneur et de précepteur. En latin, comme en espagnol on a besoin de deux mots différents pour rendre ces deux concepts: dominus et magister, et amo et maestro. Tout en respectant la concision de la formule d'Hélöise, la traduction française l'enrichit involontairement. À défaut du texte latin, c'est le mot servante qui, dans le doute, détermine le choix de ámo pour traduire maître, pour ne pas détruire le parallélisme par lequel Héloïse prétend souligner la relation entre les deux membres de ce couple. De toute façon, le texte latin reste toujours le plus succinct - il n'y a qu'à compter les mots. Et il le deviendra encore plus dans les 
suscriptions de la troisième et de la cinquième lettres, ce qui augmentera la difficulté de leur traduction, comme on verra par la suite :

Latin: Unico suo post Christum unica sua in Christo.

Français : À celui qui est tout pour elle après Jésus-Christ, celle qui est toute à lui en JésusChrist.

Espagnol: A aquél que es todo para ella después de Jesucristo, la que es toda suya en Jesucristo.

Le transvasement ne se fait pas sans perte. On ne peut pas empêcher que la force prête à éclater, comprimée dans la formule latine ne cherche à échapper au moment de la version. É. Gilson ${ }^{4}$ traduit plus fidèlement: «À son unique après le Christ, celle qui est son unique dans le Christ», ce qui en espagnol aurait donné littéralement: «A su único después de Cristo, la que es su única en Cristo.» Laide et fidèle à la lettre, mais peut-être pas à l'esprit, c'est-à-dire, à l'énorme dévotion d'Héloïse qu'elle enferme. Et d'ailleurs, ces traductions sont, en réalité des explications de la formule d'Héloïse, surtout celle d'O. Gréard; si je l'ai préférée c'est parce qu'elle passait mieux en espagnol. Mais arrêtons-nous un peu pour examiner l'énigmatique raccourci de la dernière lettre d'Hélö̈se et ses traductions :

Latin : Domino specialiter, sua singulariter.

Français: À son souverain maître, sa servante dévouée.

Espagnol: A su amo soberano, su devota sierva.

Énigmatique pour nous, mais pas pour Abélard qui fut son professeur de logique. En effet, pour lui, explique É. Gilson ${ }^{5}$, le sens de cet «étonnant et intraduisible raccourci» est clair: «elle appartient au Seigneur comme rentrant dans l'espèce des religieuses, mais comme individu, c'est à Abélard qu'elle appartient». Dans la quatrième lettre, Abélard avait tenté de lui faire comprendre qu'elle était désormais l'épouse du Christ, qu'elle devait oublier le passé. Par la suscription de sa réponse, Hélö̈se arrive à lui exprimer encore une fois son adoration, mais sans en parler. Suivant l'explication de É. Gilson, j'aurais pu traduire littéralement en espagnol: «A Dios específicamente, suya individualmente.» Ce qui sent trop l'esprit syllogistique. La traduction d'O. Gréard, encore une fois plus fidèle à l'esprit qu'à la lettre, nous renseigne, au premier coup d'œil, sur les sentiments qu'Héloïse n'a pas osé exprimer ouvertement, mais elle atténue l'éclat de son talent pour les faire tenir dans une formule si laconique. Pour lui, Héloïse est avant tout, malgré son intelligence et son érudition, une femme amoureuse. C'est aussi ce qu'elle même ne se lasse pas de répéter. Je me suis donc rendue à l'enchantement de l'aura poétique qui enveloppe la passion et qui émane de la traduction d'O. Gréard. Que ceux qui estiment qu'il y a là haute trahison «qu'ils se prennent à leurs Filozofes qui ont estimé (...) sagesse estre sans passions»; et sans passions, point de poésie.

Je me plaçai aussi sous le protection d'Octave Gréard pour choisir le registre de translation. Le saut le plus dangereux, - sept siècles en une enjambée - il l'avait déjà fait. Il ne me restait qu'à parcourir un bout de chemin pour terminer le travail de rapprochement de cette réalité si lointaine du lecteur mexicain d'aujourd'hui.

D'abord, on ne doit pas oublier qu'on ne se trouve pas ici en présence d'un roman épistolaire, mais face à de vraies lettres, écrites par deux vrais correspondants, et qu'en dehors de l'inextricable réseau de liens qui les unirent et de toute contrainte stylistique de l'époque, chacun a une manière de s'exprimer qui lui est propre. En outre, ce recueil comprend deux types de lettres: d'amour et de direction. Cette dénomination, traditionnelle, n'est pas arbitraire : il y a là deux sujets différents, donc, deux tons différents nettement perçus, surtout dans le discours d'Hélö̈se. Il faut faire entendre ces deux voix, faire 
attention à ces changements de ton pour ne pas les étouffer, pour éviter que la voix du traducteur ne se détache en solo. Ces lettres sont, d'ailleurs, parsemées de citations de la Bible, des pères de l'Église et des auteurs classiques. Invoquer une «autorité» à l'appui d'une thèse était, au XII ${ }^{e}$ siècle, la conditio sine qua non de tout écrit sérieux. Héloïse et Abélard, érudits tous les deux, respectent cette exigence. Par conséquent, on ne peut pas ignorer ces particularités et essayer d'estomper, pour ainsi dire, les contours de ces éléments étrangers intégrés à leur écriture sans la trahir. Encore une fois, c'est $O$. Gréard qui s'est chargé de résoudre ces problèmes. S'est-il posé les mêmes questions que moi? Il ne le dit pas dans sa préface. Toujours est-il qu'il a craint, lui aussi, en traducteur conscient de sa responsabilité, de ne pas avoir réussi à exprimer toute la grandeur de ces lettres. Pour ma part je ne suis pas certaine de ne pas avoir trahi, malgré moi, Héloïse, Abélard et Octave Gréard.

Nous donnons ensuite le texte original d'un paragraphe tiré de la deuxième lettre d'Hélö̈se et de ses traductions en français et en espagnol, ce qui permettra d'apprécier le degré de perte éprouvé par l'original à son passage d'une langue à une autre :

Sed haec quidem amaritudo verae poenitentiae quam rara sit beatus diligenter attendens Ambrosius: "Facilius inquit, inveni qui innocentiam servaverunt, quam qui poenitentiam egerum.s In tantum vero illae, quas pariter exercuimus, amatium voluptates dulces mihi fuerunt, ut nec displicere mihi, nex vix a memoria labi possint. Quocunque loco me vertam, semper se oculis meis cum suis ingerunt desideriis. Nec etiam dormienti suis illusionibus parcunt. Inter ipsa missarum solemnia, ubi purior esse debet oratio, obscena earum voluptaum phantasmata ita sibi penitus misserrimam captivant animam, ut turpitudinibus illus magis quam orationi vacem. Quae quum ingemiscere debeam de comissis, suspiro potius de amissis.

Mais cette amertume du vrai repentir est bien rare, et saint Ambroise en fait la remarque: «J'ai trouvé, dit-il, plus de cœurs qui ont conservé leur innocence que de cours qui ont fait pénitence.» Quant à moi, ces voluptés de l'amour que nous avons goûtées ensemble m'ont été si douces, que je ne puis m'empêcher d'en aimer le souvenir, ni l'effacer de ma mémoire. De quelque côté que je me tourne, elles se présentent, elles s'imposent à mes regards avec les désirs qu'elles réveillent; leurs illusions n'épargnent même mon sommeil. Il n'est pas jusqu'à la solennité de la messe, là où la prière doit être si pure, pendant laquelle les licencieuses images de ces voluptés ne s'emparent si bien de ce misérable cour, que je suis plus occupée de leur turpitude que de l'oraison. Je devrais gémir des fautes que j'ai commises, et je soupire après celles que je ne puis plus commettre.

Pero la amargura del verdadero arrepentimiento es muy rara, y San Ambrosio lo hace notar: "He encontrado, dice, más corazones que han conservado la inocencia que corazones que han hecho penitencia». En cuanto a mí, esos placeres del amor que juntos saboreamos me fueron tan dulces, que no puedo evitar su recuerdo, ni borrarlo de mi memoria. Para cualquier lado que me vuelva, se presentan, se imponen a mis miradas con los deseos que despiertan; sus ilusiones ni siquiera perdonan mi sueño. Hasta en la solemnidad de la misa, alli donde la plegaria debe ser tan pura, las licenciosas imágenes de estos placeres se apoderan de tal manera de este miserable corazón, que estoy más ocupada de sus bajezas que de la orazión. Debería gemir por las faltas que cometí y suspiro por las que ya no puedo cometer.

Ce que je viens de décrire dans ces pages n'est peut-être qu'un pâle reflet de «cet effort ingrat accompli consciencieusement sans espoir d'argent ni de gloire ${ }^{\epsilon_{\gg}}$ qu'on appelle généralement la pratique de la traduction littéraire. Celle-ci n'étant que mon violon d'Ingres, je n'ai pas osé parler de ce que je n'ai connu qu'à demi: les salaires de famine, les contrats draconiens, le mince prestige social accordé à cette profession. Traitement injuste qui contredit la nature même d'un métier dont l'exercice demande, au moins, la maîtrise de deux langues, des études supérieures spécialisées, les vertus et la 
discipline d'un trappiste et encore, le génie d'un écrivain pour éviter «ce jargon abominable qu'on appelle le traduit -du $u^{7} \gg$ et développer un style beau et fidèle à l'original qu'on n'acquiert pas par la seule observation des règles ou la lecture des œuvres théoriques, mais au prix d'un labeur patient et opiniâtre dont le succès - on ne le sait que trop sera toujours relatif.

\section{NOTES :}

1. «Les yeux ouverts», L'Art de traduire, Paris, éditions du Centurion, 1980.

2. Gérard Guillot, Louise Labé, Seghers, p. 211.

3. GRÉARD, O. (traduction nouvelle précédée d'une préface par): Lettres complètes d'Abélard et d'Héloise, Paris, Garnier Frères.

4. (1984) : Hêloïse et Abélard, Paris, Vrin, coll. «Librairie philosophique», p. 125.

5. Op. cit.

6. TOURNIER, M. : Le Vent Paraclet, Paris, Folio, p. 164.

7. Op. cit. 